\title{
Measuring sea-ice concentration and floe-size distribution by image processing
}

\author{
Ken-IChiro Muramoto, Kohki MatsuUra, \\ Department of Electrical and Computer Engineering, Faculty of Technology, Kanazawa University, Kanazawa 920, Japan \\ TATSUO ENDOH \\ Institute of Low Temperature Science, Hokkaido University, Sapporo 060, Japan
}

\begin{abstract}
A technique for analyzing sea-ice concentration and floe-size distribution by means of image processing is proposed. The sea ice was photographed by video camera from the ship. The technique proposed for analyzing sea-ice images can be applied on line or off line. In on-line analysis, sea-ice images photographed by a video camera are digitized in real time. Ice concentration is calculated by summing ice pixels of each row of a digital image and ice shape can be obtained roughly by a composition of each row. In off-line analysis, sea-ice images recorded to a video recorder are processed. Both ice shape and ice concentration can be obtained accurately by analyzing predetermined square regions of an image. Although the off-line method requires more time to calculate, it is useful for detailed analysis of regional ice properties. Computations of ice concentration and floe-size distribution are performed using images obtained between Breid Bay and Syowa Station in 1988.
\end{abstract}

\section{INTRODUGTION}

Measurement of the seasonal cycle and interannual variations of Antarctic sea ice is important for investigations of global climate (Gordon and Taylor, 1975; Sturman and Anderson, 1985; Allison, 1989). Monitoring of sea ice is also important for ship navigation. While satellite data give a great amount of information about ice conditions (Comiso and Zwally, 1982; Zwally and others, 1983; Sturman and Anderson, 1985), there still remains a need for in situ validations (Comiso and others, 1984; Andreas and Makshtas, 1985; Jacka and others, 1987). However, visual observation of ice conditions is often difficult and is always laborious, and there is a limit to the validity of estimates of ice concentration which rely upon qualitative observation (Jacka and others, 1987; Allison, 1989).

We propose a system which automatically measures the ice concentration and floe-size distribution using a computer and image digitizer. Preliminary experiments to test the system have been carried out in ice-covered water in the Southern Ocean.

\section{SYSTEM CONFIGURATION}

The sea ice was photographed by a video camera mounted on board the icebreaker Shirase as shown in Figure 1. The camera was located at the upper steering house pointing at an angle of $10^{\circ}$ downward from the horizon. The image taken by this camera is dealt with in two ways: analysis of image on line and storage of the image for analysis off line. The image is divided into $256 \times 256$ pixels on line or off line using an image digitizer. At each pixel location, the image brightness is quantified into 256 grey levels.

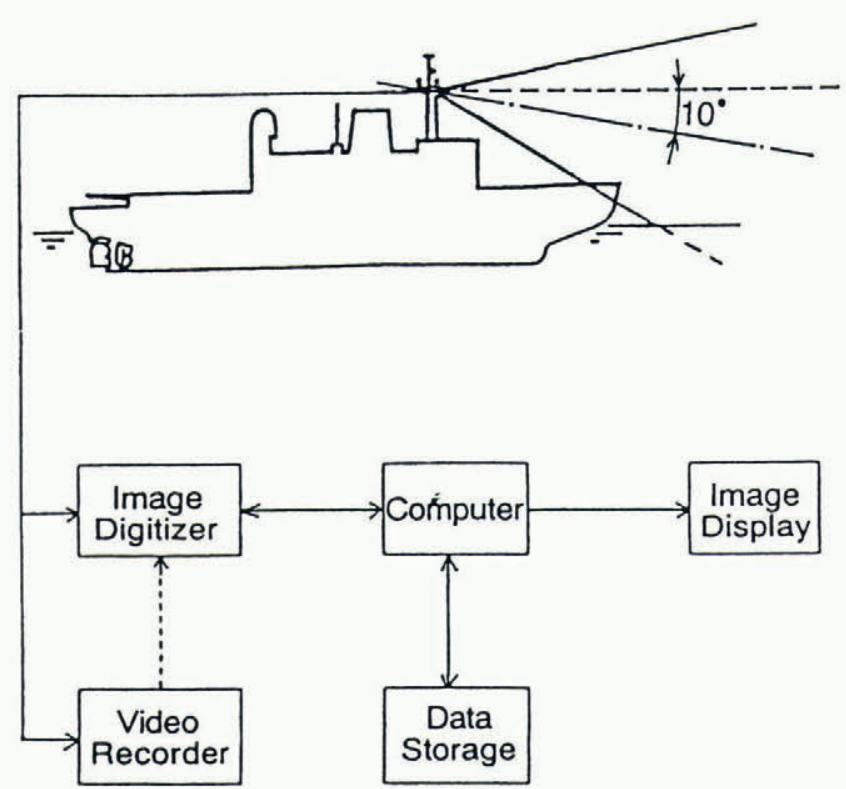

Fig. 1. System configuration for photographing sea ice. The camera was located at the upper steering house with an angle of $10^{\circ}$ downward from the horizon. 
For the on-line analysis, sea-ice images are digitized in real time. Ice concentration is calculated by summing ice pixels of each row of the digital image and ice shape can be obtained roughly by composition of the fraction of ice in each row.

For the off-line analysis, video recorded sea-ice images are processed. Both ice shape and concentration can be obtained accurately by analyzing predetermined square regions of the images.

\section{ON-LINE ANALYSIS}

In order to measure the sea-ice concentration and shape in real time, one horizontal line (row) at the same position within each digital image is analyzed at predetermined time intervals as shown in Figure 2A. Since the sea-ice image is approaching the ship during navigation, orthographic projection of ice-covered water is obtained by patching the rectangular area that is composed of horizontal length of a single row and
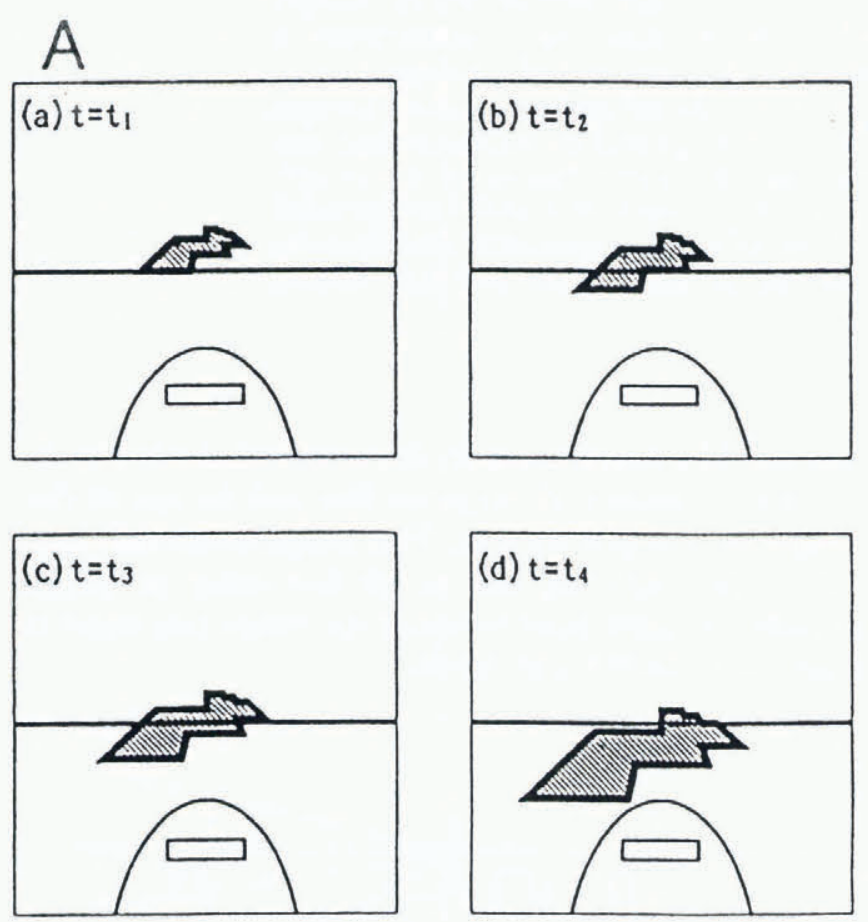

B

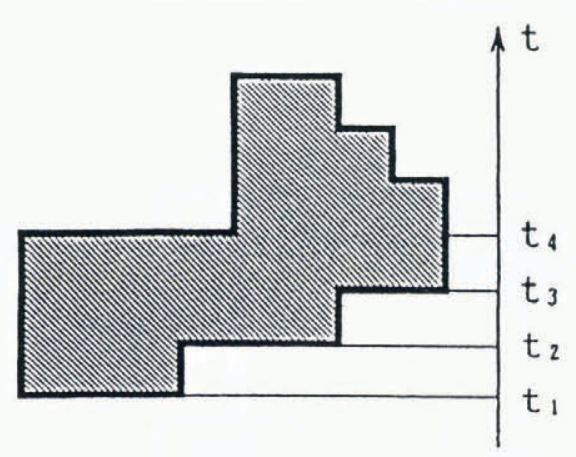

Fig. 2. Schema of composition of sea ice. The floe representation, $B$, is composed of the fraction of ice on each row in $A$. Since images are sampled at fixed finite intervals, they are composed of roughly rectangular arrays of pixels.
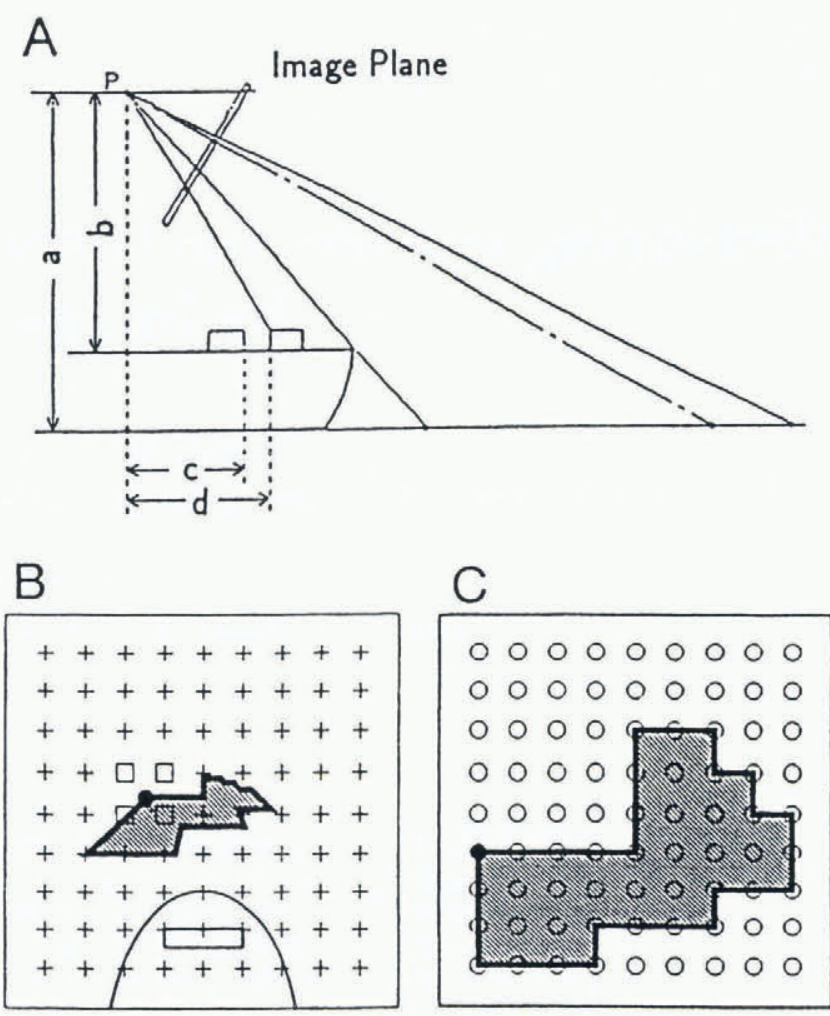

Fig. 3. Schema of geometric transformation. A, spatial relation between the sea water and its projection in the image plane. Using known lengths $(a-d)$ and angles, geometric transformation is performed. $B$, oblique projection. $C$, orthographic projection transformed from trapezoid region shown in $B$ on oblique projection. The grey levels of input image $B$ are transferred to the output image $C$ pixel by pixel. If an output pixel $(\bullet)$ maps to a position between four input pixels ( $\square$ ), then its grey level is divided among the four input pixels according to the interpolation rule.

expected vertical length (Figure 2B). The vertical length is determined by the speed of the ship. The sea-ice concentration is calculated from the ratio of ice to water for each row. To reduce the processing time, images are sampled every second, thus each floe is composed of rough rectangular arrays of pixels.

If the speed or course of the ship is changed, the shape of the floe is distorted. Although some limitations exist, ice concentration and rough floe size can be obtained in real time by this analysis.

\section{OFF-LINE ANALYSIS}

Figure $3 \mathrm{~A}$ shows the spatial relation between the sea surface and its projection in the image plane. Using known lengths and angles such as camera height, distance from camera to bow and angle of depression of the camera, a geometric transformation is performed. By this transformation, a square region of sea with a side of $180 \mathrm{~m}$ is analyzed, and each image of sea ice (Fig. 3B) is transformed to an orthographic projection (Fig. 3C). In the geometric operation, the output pixels are mapped into the input image to establish their grey level. If an output pixel falls between four input pixels, interpolation is necessary to determine the grey level of the output pixel 
(Castleman, 1979). In this process, as the distance from the ship increases, the area of a pixel increases, and the accuracy of the area of remote pixels lessens.

\section{TRANSFORMATIONS}

\section{The spatial transformation}

The general definition for a geometric operation is

$$
g(u \prime, v \prime)=f(u, v)=f[a(u, v), b(u, v)],
$$

where $f(u, v)$ is the input image and $g(u \prime, v \prime)$ is the output image as shown in Figure 4. The functions $a(u, v)$ and $b(u, v)$ uniquely specify the spatial transformation. In the input image $f(u, v)$, the grey-level values are defined only at integral values of $u$ and $v$. However, this equation will in general dictate that the grey level value for $g(u \prime, v \prime)$ be taken from $f(u, v)$ at non-integral coordinates. As mentioned previously, the output pixels map to fractal position in the input image, generally falling in the space between four input pixels. Interpolation is necessary to determine the grey level of the output pixel.

\section{Nearest-neighbor transformation}

The simplest interpolation scheme is the so-called zeroorder or nearest-neighbor interpolation. In this case, the grey level of the output pixel is taken to be that of the input pixel nearest to the position to which it maps as shown in Figure 5A. This is computationally simple and produces acceptable results in many cases. However, nearest-neighbor interpolation can introduce artifacts in images containing fine structure whose grey level changes significantly over one unit of pixel spacing.

\section{Bilinear transformation}

First-order interpolation produces more desirable results with a slight increase in programing complexity and execution time. In Figure 5B, let $f\left(u_{0}, v_{0}\right)$ be a function of two variables that is known at $(u \prime, v \prime),(u \prime+1, v \prime)$, $(u \prime, v \prime+1)$ and $(u \prime+1, v \prime+1)$, which are the vertices of the unit square. Suppose we desire to establish the value of $f\left(u_{0}, v_{0}\right)$ at an arbitrary point inside the square by interpolation through the four known values. We shall do

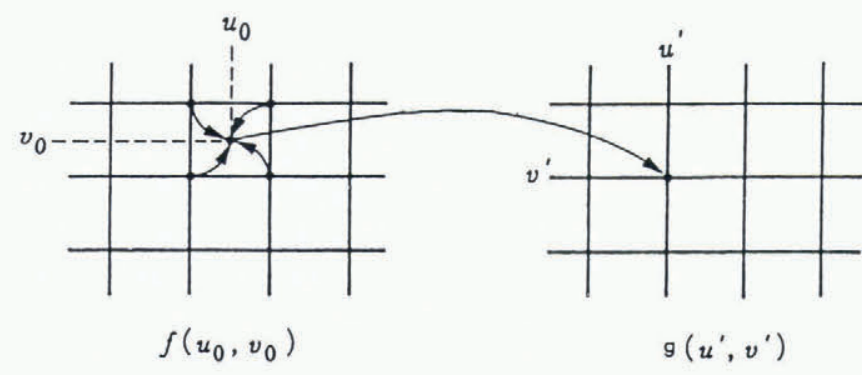

Fig. 4. The spatial relationship between points in the input image and points in the output image. The output pixels are mapped into the input image. If an output pixel maps to a position between four input pixels, then interpolation is necessary to determine the grey level of the output pixel.
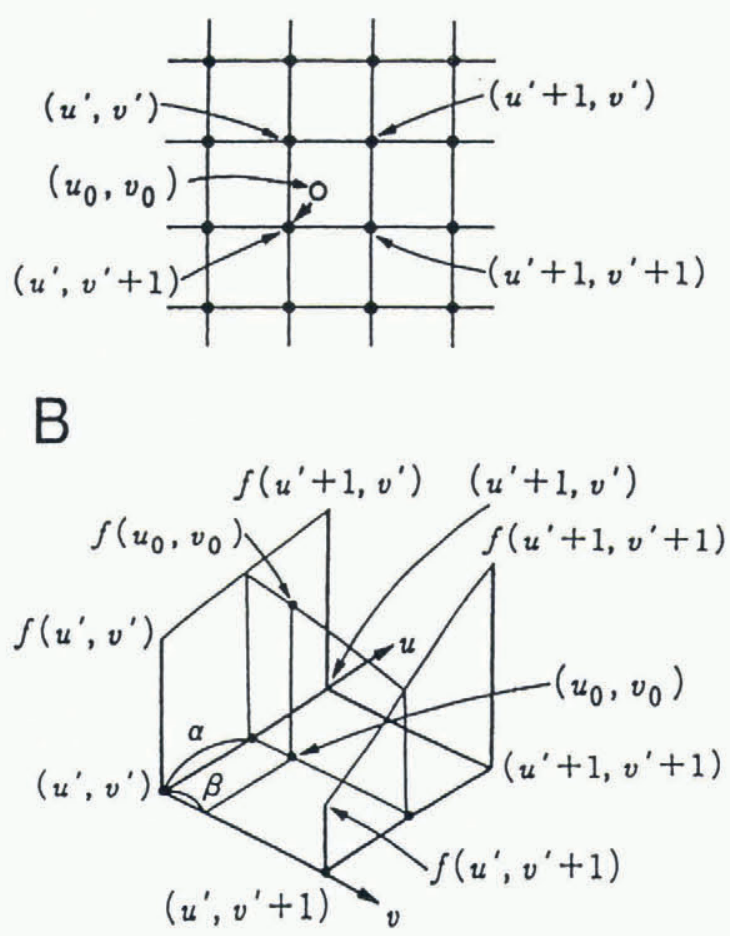

Fig. 5. Grey-level interpolation. A, nearest-neighbor interpolation. In this case, the grey level of the output pixel is taken to be that of the input pixel nearest to the position to which it maps. $B$, bilinear interpolation. In this case, the grey level of the output pixel is determined by the bilinear equation using the known values at four corners.

so by fitting a hyperbolic paraboloid, defined by the bilinear equation

$$
\begin{aligned}
f\left(u_{0}, v_{0}\right)= & f(u \prime, v \prime)(1-\alpha)(1-\beta)+f(u \prime+1, v \prime) \alpha(1-\beta) \\
& +f(u \prime, v \prime+1)(1-\alpha) \beta \\
& +f(u \prime+1, v \prime+1) \alpha \beta .
\end{aligned}
$$

An example of sea-ice images with transformations

Figure 6 compares bilinear with nearest-neighbor transformation. In these photographs, white areas indicate ice and black areas indicate water. The trapezoid region of ice-covered water in Figure 6A, transformed to an orthographic projection by nearest-neighbor and bilinear interpolation procedures, is shown in Figure 6B and D. To separate ice from sea water, the transformed images are converted to a binary image by a suitable grey-level threshold, as shown in Figure 6C and E. The threshold level was determined using a grey-level histogram of the image at the beginning of the analysis, and this level was fixed. Although we examined some points for the effect of threshold level, almost all separated satisfactorily except the case of back lighting from the sun. In Figure 6C a sawtooth effect appears at some of the edges. In Figure 6E desirable result is obtained. The ice concentration is calculated by summing ice pixels over the $180 \mathrm{~m} \times 180 \mathrm{~m}$ area to get a regional measure. Floe size could be determined only for floes completely contained 

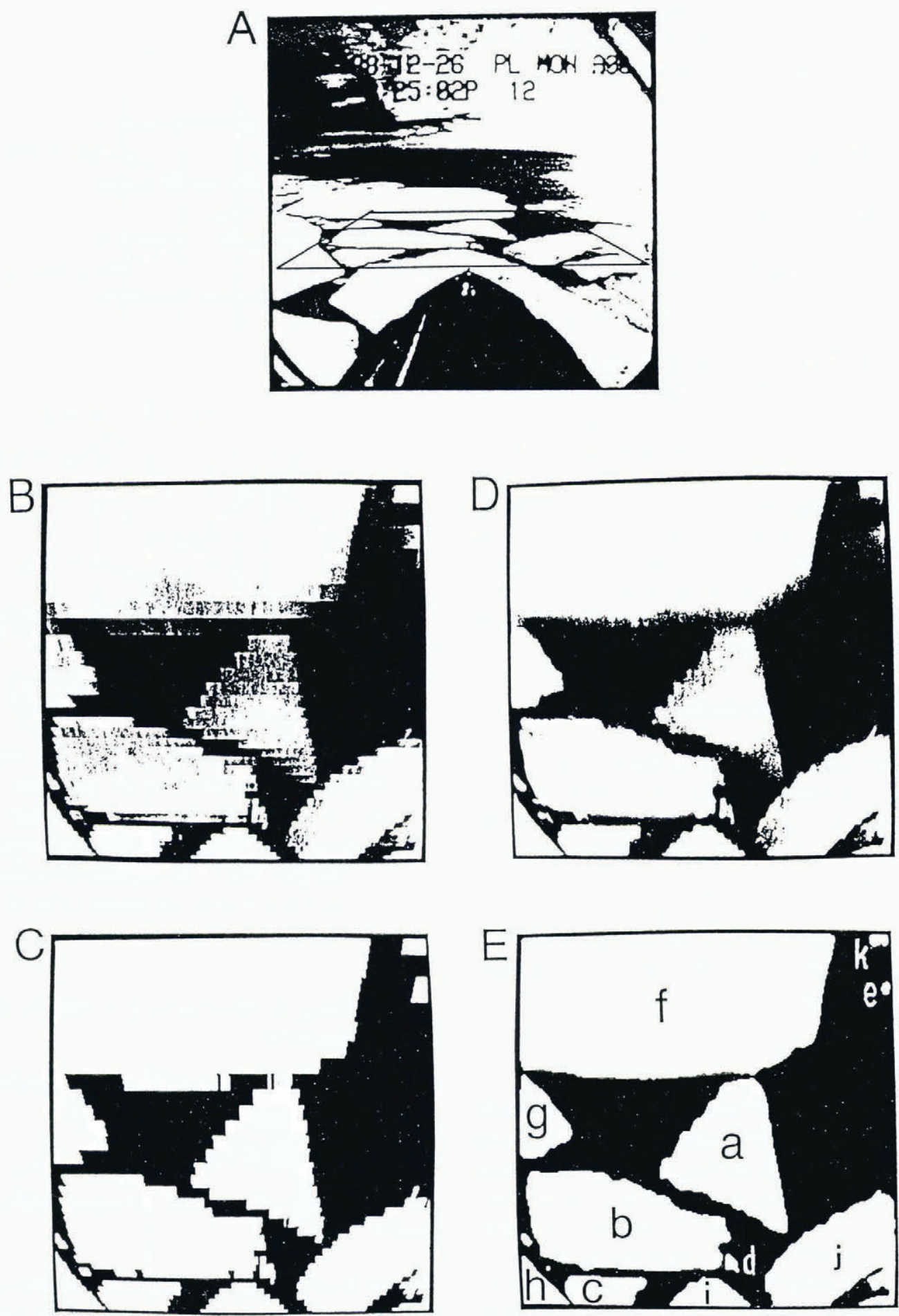

Fig. 6. Comparison of nearest-neighbor and bilinear interpolation. $A$, an original image. The trapezoid region on icecovered water was transformed to orthographic projection by nearest-neighbor $(B)$ and bilinear $(D)$ interpolation. $C, E$, binary image of $B$ and $D$, respectively.

within an image. Though bilinear transformation requires much calculation, this method is useful for detailed analysis of regional ice properties.

\section{RESULTS}

The sea-ice image was recorded between Fremantle, Australia, and Syowa Station in 1988 by T.Endoh, a member of the 30th Japanese Antarctic Research Expedition (JARE-30). The cruise track of the icebreaker Shirase from Breid Bay to Syowa Station is shown in Figure 7.
On-line analysis was performed at predetermined time intervals using the images photographed during navigation. The ice concentration and shape were analyzed every second as shown in the example at Figure 8, and the data were stored. Figure 9A shows changes of ice concentration estimated every $5 \mathrm{~s}$ during the first $24 \mathrm{~h}$ from Breid Bay to Syowa Station. Figure 9B depicts $10 \mathrm{~min}$ running means of the ice concentration. These graphical records are useful to examine the general tendency of ice concentration during the observation. Because the vessel navigates to find the easiest passage, the ice concentration may be biased towards a lower limit. The differences between ice concentrations along 


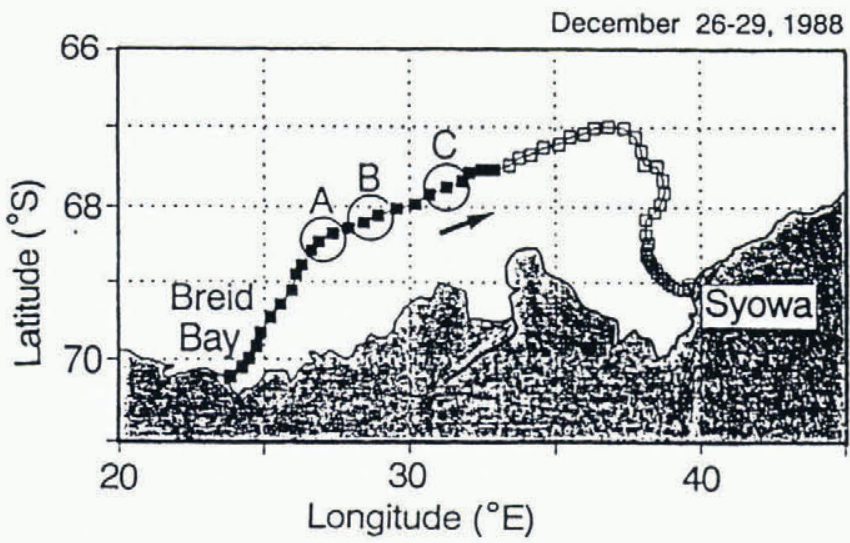

Fig. 7. Cruise track of the icebreaker Shirase every hour (solid and open square) from 26-29 December 1988.

\section{A}

Ice shape

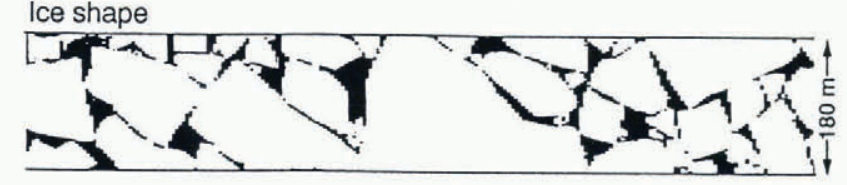

100 Concentration [\%]

B

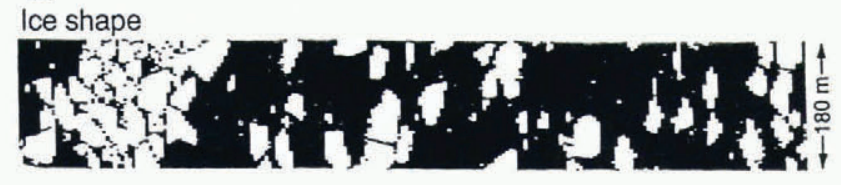

100 Concentration [\%]

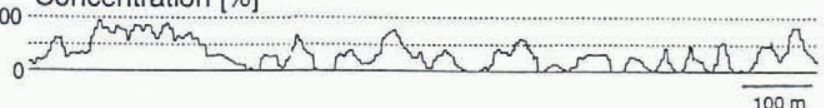

Fig. 8. Examples of ice concentration and shape resulting from analysis every second. Mean ice concentrations during 5 min intervals shown were $81.6 \%$ in $A$ and $29.0 \%$ in $B$.

the route and in neighboring regions must be identified by alternative methods, such as by observation from helicopter or by remote sensing.

Table 1 shows the 11 floe sizes of Figure 6E. While floes a-e were surrounded by water on the image display, allowing calculation of floe area, floes $f-k$ were not. Figure 10 shows the floe-size distributions calculated for the areas A, B and C of Figure 7. In some cases, the ice concentrations had almost the same value but the distributions of floe size were different. Figure $10 \mathrm{~A}$ and $\mathrm{C}$ shows an example of such a case: a large area of ice but a small number of floes in Figure $10 \mathrm{~A}$ and vice versa in Figure 10C.

During on-line analysis, the data from time series analysis of the ice concentration will be useful for investigation of long-term variations (Fig.9). In this analysis, however, the ice shape cannot be obtained with accuracy. In the off-line analysis, both ice shape and ice concentration can be accurately obtained by analyzing predetermined square regions of an image (Figs 6 and 10). Although the off-line method requires more time to calculate, it is useful for detailed analysis of regional ice
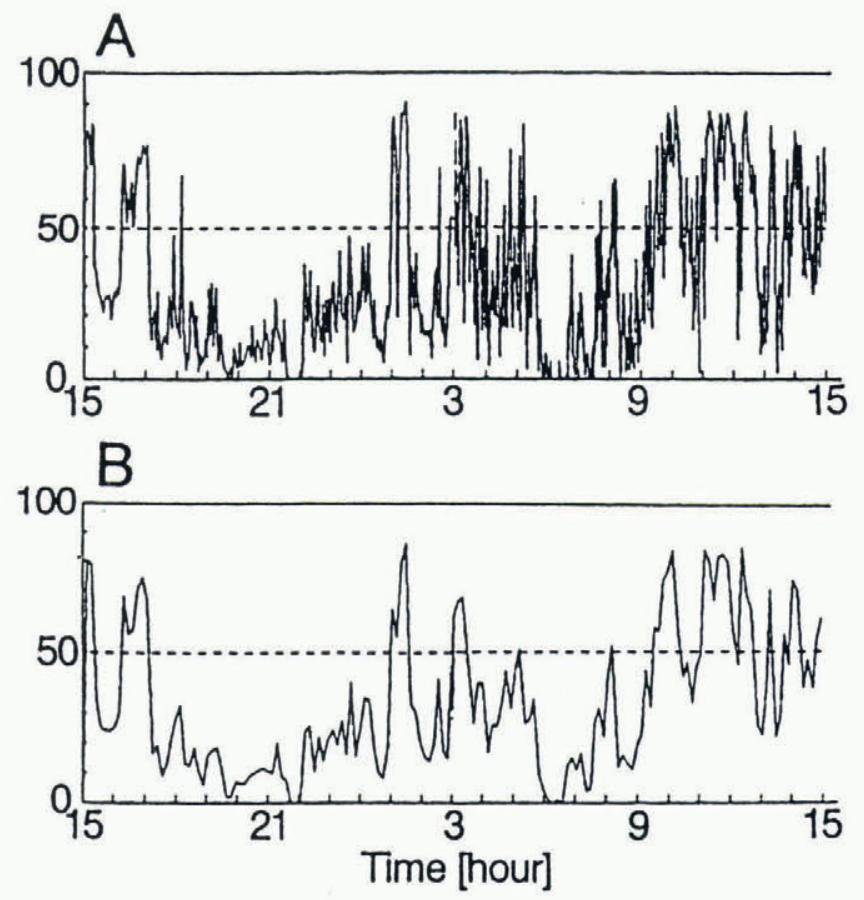

Fig. 9. Changes of ice concentration during the first $24 \mathrm{~h}$ from Breid Bay to Syowa Station (solid squares shown in Fig. 7). A, estimated every $5 \mathrm{~s} ; \mathrm{B}, 10$ min running mean.
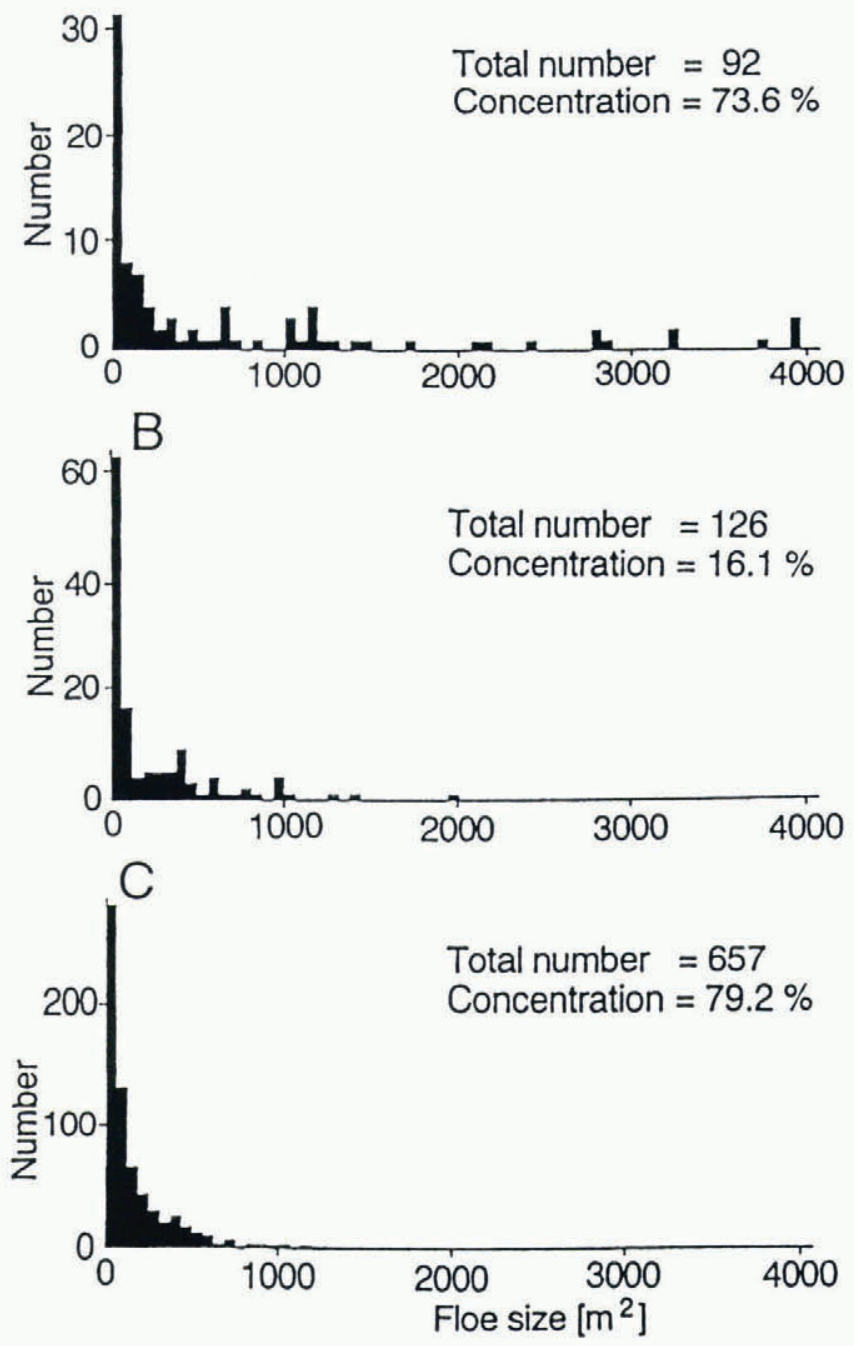

Fig. 10. Floe-size distribution for the three areas $A, B$ and $C$ of Figure 7. Although the ice concentrations had almost the same value in $A$ and $C$, distributions of floe size were different. 
Table 1. Floe size of Figure $6 E$

\begin{tabular}{rrrr}
\hline & $\mathrm{m}^{2}$ & & $\mathrm{~m}^{2}$ \\
\hline $\mathrm{a}$ & 2418.3 & $\mathrm{f}$ & 9869.9 \\
$\mathrm{~b}$ & 3638.2 & $\mathrm{~g}$ & 605.6 \\
$\mathrm{c}$ & 680.6 & $\mathrm{~h}$ & 597.7 \\
$\mathrm{~d}$ & 37.8 & $\mathrm{i}$ & 968.8 \\
$\mathrm{e}$ & 10.3 & $\mathrm{j}$ & 3020.5 \\
& & $\mathrm{k}$ & 42.3 \\
\hline
\end{tabular}

Concentration $=67.6 \%$

properties. It follows from these results that choice of analysis depends on purpose.

\section{CONCLUSION}

The system for analyzing sea-ice concentration and floesize distribution offers several practical advantages. Chief among these are: (1) All the hardware components are commercially available. (2) The system is easy to use. (3) The software can easily be adapted to an image photographed from another camera position. (4) Online or off-line analysis can be utilized depending on the purpose. (5) Furthermore, this technology will be easily applied to images obtained in other ways such as from helicopters or aircraft.

\section{ACKNOWLEDGEMENTS}

The authors thank M.Kosugi for development of software.

\section{REFERENCES}

Allison, I. 1989. The East Antarctic sea ice zone: ice characteristics and drift. Geogr. F., 18(1), 103-115.

Andreas, E. L. and A.P. Makshtas. 1985. Energy exchange over Antarctic sea ice in the spring. $\mathcal{J}$. Geophys. Res., 90(C4), 7199-7212.

Castleman, K. R., ed. 1979. Digital image processing. Englewood Cliffs, NJ, Prentice-Hall.

Comiso, J. C. and H.J. Zwally. 1982. Antarctic sea ice concentrations inferred from Nimbus 5 ESMR and Landsat imagery. 7. Geophys. Res., 87(C8), 5836-5844.

Comiso, J. C., S.F. Ackley and A. L. Gordon. 1984. Antarctic sea ice microwave signatures and their correlation with in situ ice observations. 7. Geophys. Res., 89(C1), 662-672.

Gordon, A. L. and H. W. Taylor. 1975. Seasonal change of Antarctic sea ice cover. Science, 187(4174), 346-347.

Jacka, T.H., I. Allison, R. Thwaites and J. C. Wilson. 1987. Characteristics of the seasonal sea ice of East Antarctica and comparisons with satellite observations. Ann. Glaciol., 9, 85-91.

Sturman, A. P. and M. R. Anderson. 1985. A comparison of Antarctic sea ice data sets and inferred trends in ice area. 7. Climate Appl. Meteorol., 24(3), 275-280.

Zwally, H.J., J. C. Comiso, C.L. Parkinson, W.J. Campell, F. D. Carsey and P. Gloersen. 1983. Antarctic sea ice, 1973-1976: satellite passive-microwave observations. Washington, DC, National Aeronautics and Space Administration. (NASA SP-459.)

The accuracy of references in the text and in this list is the responsibility of the authors, to whom queries should be addressed. 